\title{
Simultaneous multiview capture and fusion improves spatial resolution in wide-field and light-sheet microscopy: supplementary material
}

\author{
Yicong Wu, ${ }^{1,}{ }^{*}$ Panagiotis Chandris, ${ }^{1}$ Peter W. Winter, ${ }^{1}$ Edward Y. Kim, ${ }^{2}$ \\ Valentin Jaumouillé, ${ }^{3}$ AbHishek Kumar, ${ }^{1}$ Min Guo, ${ }^{1,4}$ Jacqueline $M$. \\ Leung, ${ }^{5}$ Corey SMith, ${ }^{6}$ Ivan Rey-Suarez, ${ }^{1}$ Huafeng LiU, ${ }^{4}$ Clare $M$. \\ Waterman, ${ }^{3}$ Kumaran S. RamamurthI, ${ }^{2}$ Patrick LaRIVIERe, ${ }^{6}$ AND HaRI \\ SHROFF ${ }^{1}$
}

${ }^{1}$ Section on High Resolution Optical Imaging, National Institute of Biomedical Imaging and Bioengineering, National Institutes of Health, Bethesda, MD 20892, USA.

${ }^{2}$ Laboratory of Molecular Biology, National Cancer Institute, National Institutes of Health, Bethesda, MD 20892, USA.

${ }^{3}$ Cell Biology and Physiology Center, National Heart, Lung, and Blood Institute, National Institutes of Health, Bethesda, MD 20892, USA.

${ }^{4}$ State Key Lab of Modern Optical Instrumentation, College of Optical Science and Engineering, Zhejiang University, Hangzhou 310027, China.

${ }^{5}$ Department of Biology, Indiana University Bloomington, Bloomington, IN 47405, USA.

${ }^{6}$ Department of Radiology, University of Chicago, Chicago, IL 60637, USA.

*Corresponding author: yicong.wu@nih.gov

Published 11 August 2016

This document provides supplementary information to "Simultaneous multiview capture and fusion improves spatial resolution in wide-field and light-sheet microscopy," http://dx.doi.org/10.1364/optica.3.000897. This supplement consists of supplementary video captions, a supplementary note, and 16 supplementary figures.

(C) 2016 Optical Society of America

http://dx.doi.org/10.1364/optica.3.000897.s001

\section{Supplementary Video Captions}

Visualization 1. Comparative 3D projections of triple- and bottom-view wide-field imaging of Bacillus subtilis cells expressing SpoVM-GFP. Also evident are $100 \mathrm{~nm}$ yellowgreen fluorescent beads used in registration. Left: tripleview imaging; Right: bottom-view imaging. The 3D projections are rotated about the $\mathrm{Y}$ axis (with respect to the coordinate system of Objective $\mathrm{C}$ ). The improvement in axial resolution provided by triple-view imaging is especially evident in 90 and 270 degree views. See also Fig. 2a.

Visualization 2. Higher magnification view of Visualization 1, showing a single Bacillus subtilis cell. Top: triple-view imaging; Bottom: bottom-view imaging. The enhanced axial resolution provided by triple- view imaging recovers the circular shape of the forespore. See also Fig. 2b-f.

Visualization 3. Comparative YZ slices of triple- and bottomview wide-field imaging of Toxoplasma gondii expressing EGFP-Tg $\beta 1$-tubulin. The yellow line indicates the coverslip surface. Top: triple-view imaging; Bottom: bottom-view imaging. Triple-view imaging preserves the axial shape of the parasites. See also Fig. 2g-l.

Visualization 4. Comparison between triple- and bottomview wide-field time-lapse imaging of a U2OS cell expressing Lck-tGFP (cyan, labeling the plasma membrane) and ERGIC3-mCherry (magenta, labelling dilated and vesiculated ER). Left: triple-view imaging; Right: bottom-view imaging. The cell was imaged every 15 seconds over 30 minutes imaging duration. Two typical YZ views with a separation of $1 \mu \mathrm{m}$ in the $\mathrm{X}$ direction are shown. The yellow 
line indicates the coverslip surface. Time is indicated as minute : second. The nuclear periphery, cell membrane, and vesicles are badly distorted in single-view imaging, and are much better resolved after triple-view deconvolution. See also Fig. 2m-p.

Visualization 5. Comparison between triple- and dual-view light-sheet imaging of F-tractin-EGFP in RAW264.7 macrophage. Maximal intensity $\mathrm{XY}$ and $\mathrm{ZY}$ projections covering the entire cell $(\sim 12 \mu \mathrm{m}$ thick) are shown. Top: triple-view imaging; Bottom: dual-view imaging. The cell was imaged every 15 seconds for a total duration of 38 minutes, spanning 150 volumes ( 6 seconds per volume for 120 planes at $0.5 \mu \mathrm{m}$ inter-plane spacing, then introducing a 9 seconds pause between acquisitions). The increased lateral resolution in triple-view imaging allows better observation of actin-based protrusions. See also Fig. 4a.

Visualization 6. The same sample as Visualization 4, showing two XY planes with an axial separation of $3 \mu \mathrm{m}$. Top: triple-view imaging; Bottom: dual-view imaging. Actin ruffles and filopodia are better resolved in triple- than dualview imaging. See also Fig. 4b-c.

Visualization 7. Comparative dual-color triple-view and dual-view light sheet volumetric time lapse imaging of GFPRas and mCherry-Rab8 in a U20S cell. Maximum intensity XY projections over an axial range of $1 \mu \mathrm{m}$ are shown. Left: triple-view imaging; Right: dual-view imaging. The cells was imaged every 30 seconds over more than 1 hour, spanning 140 volumes (16 seconds to acquire one dual-color volume over 320 planes at $0.5 \mu \mathrm{m}$ inter-plane spacing, and 14 seconds inter-volume pause). Triple-view imaging provides better visualization of fine structures such as filopodia, reticular structures within the plasma membrane, and intracellular vesicles and endosomes. See also Fig. 4d.

Visualization 8. Higher magnification view of sample in Visualization 7, showing a typical XY plane at $\mathrm{z}=1 \mu \mathrm{m}$ above the coverslip. Left: triple-view imaging; Right: dualview imaging. See also Fig. 4e-f.

Visualization 9. Comparative triple- and dual-view timelapse light-sheet imaging of mitochondria labeled with Mitotracker Red. Top: triple-view imaging; Bottom: dualview imaging. Maximum intensity XY projections over $1.5 \mu \mathrm{m}$ are shown. Cells were sampled every 30 seconds over 20 minute imaging duration, spanning 60 volumes $(10$ seconds to acquire one volume with 200 planes at $0.5 \mu \mathrm{m}$ inter-plane spacing, and 20 seconds inter-volume pause). The nonhomogenous, 'beads on a string' staining of mitochondria is better resolved in triple-view imaging. See also Fig. 4m-o.

\section{Supplementary Note 1}

In conventional dual-view inverted selective plane illumination microscopy (diSPIM), two identical, orthogonal objectives are used for sequential excitation and detection. There is thus a strong steric constraint: the two objectives must not collide with each other. We typically employ two 0.8 NA objectives, the highest symmetric combination that is commercially available. Therefore, the lateral and axial resolutions are both limited to $\sim 330 \mathrm{~nm}$ after joint deconvolution. However, as shown in Fig. 1, fluorescence emitted in the direction of the coverslip is not captured in diSPIM, and here we propose that a higher NA lens beneath the coverslip could in principle boost the resolution of diSPIM system without compromising acquisition speed by simultaneously imaging and fusing this normally unused view with the conventional diSPIM upper views. Since the only physical constraint on the lower objective is that it must fit beneath the coverslip, the NA can be higher than in normal diSPIM. Here we used a $1.2 \mathrm{NA}$ water immersion lens to minimize spherical aberration when imaging aqueous samples.

However, this lower objective is not perpendicular to the light sheets created in diSPIM, so at any particular instant, most of the light sheet is out-of-focus in the lower view. In order to capture the high quality, in-focus signals within the light sheet, we vertically scanned the lower objective and synchronized the vertical scan with a virtual confocal slit to filter out much of the out-of-focus signal. With this method, the dwell time at each $\mathrm{z}$ position is very short. For example, when covering a $10 \mu \mathrm{m}$ thick sample, the effective exposure time from the bottom view is only $\sim 10 \%$ of upper view (i.e., the ratio of the $\sim 1 \mu \mathrm{m}$ depth-of-focus of the 1.2 NA lens to the total sample thickness). Although the 1.2 NA lens has a larger NA, and thus a larger collection efficiency (in theory, $2.85 \mathrm{x}$ ) than the $0.8 \mathrm{NA}$ in top view (collection efficiency in Fig. S1), the smaller pixel size in the lower view offsets this enhancement $(108.3 \mathrm{~nm}$ in bottom view vs. $162.5 \mathrm{~nm}$ in the top view implies a 2.25 -fold loss in per-pixel signal intensity). Experimentally, we find that the overall signal intensity obtained from the bottom view is $\sim 1 / 7$ of each top view when imaging cellular samples. For thicker samples, the signal collected from the bottom view would be proportionately less; for example, the signal collected from the bottom view is less than $5 \%$ of the top view when imaging a $30 \mu \mathrm{m}$ nematode embryo.

In order to fuse the low signal-to-noise ratio (SNR) but high-resolution signal in the lower view to the two top views, we treat each view as having the same contribution (i.e., before deconvolution, we normalize each of the three views to its particular peak intensity), then use an additive deconvolution method to reduce noise amplification (as shown in Fig. S14) during joint deconvolution. Our deconvolution outcomes demonstrate that, despite the lower SNR of the lower view, resolution enhancement is still evident after triple-view fusion, as shown in Figs. 3-4. 


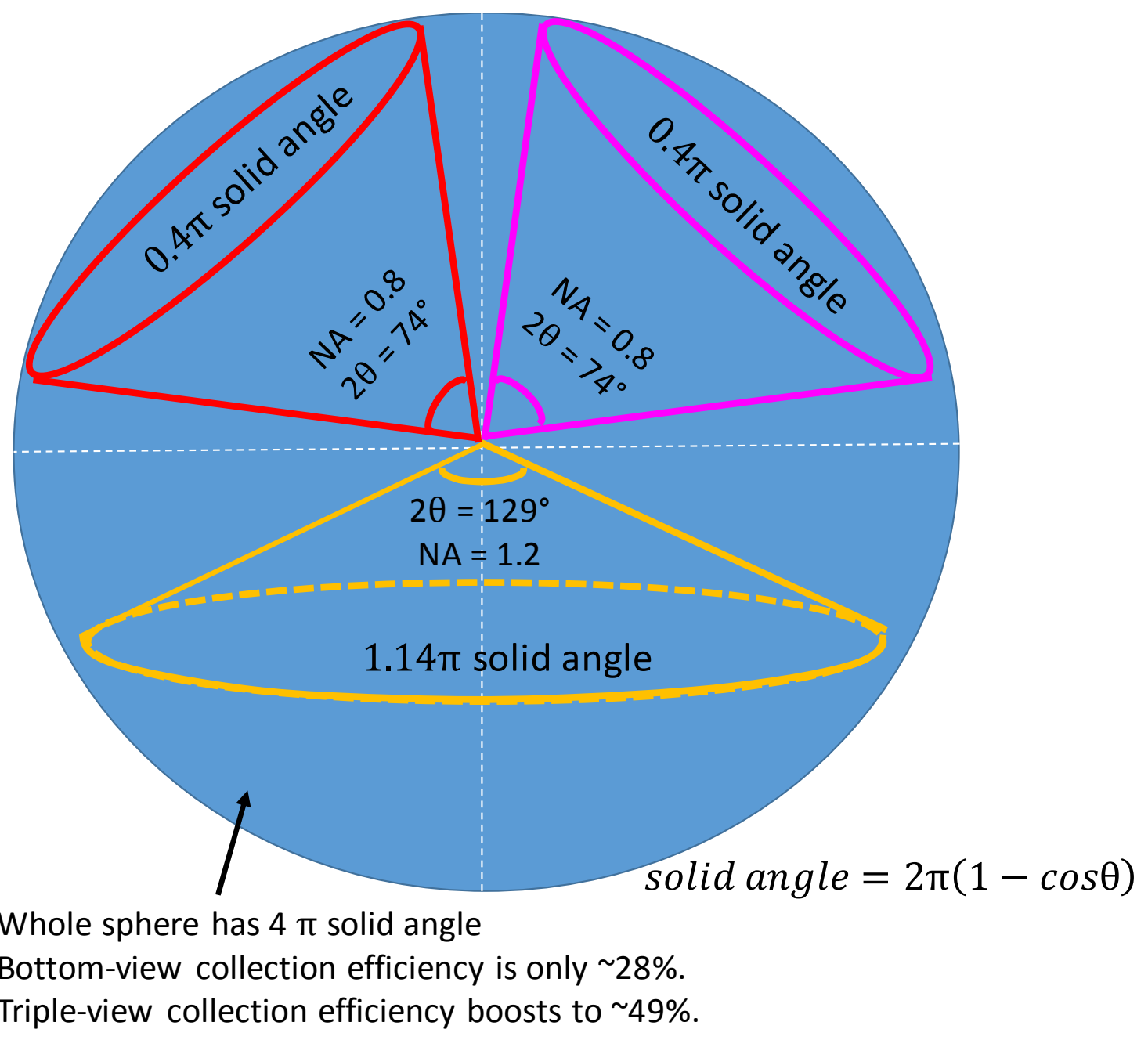

Fig. S1. Collection efficiency with objectives A, B, C. The bottom, water immersion objective has 1.2 NA and collects only 28\% of the total solid angle (4pi steradians). If the two upper, $0.8 \mathrm{NA}$ objectives are also used to simultaneously detect fluorescence, collection efficiency becomes almost half the total solid angle. 

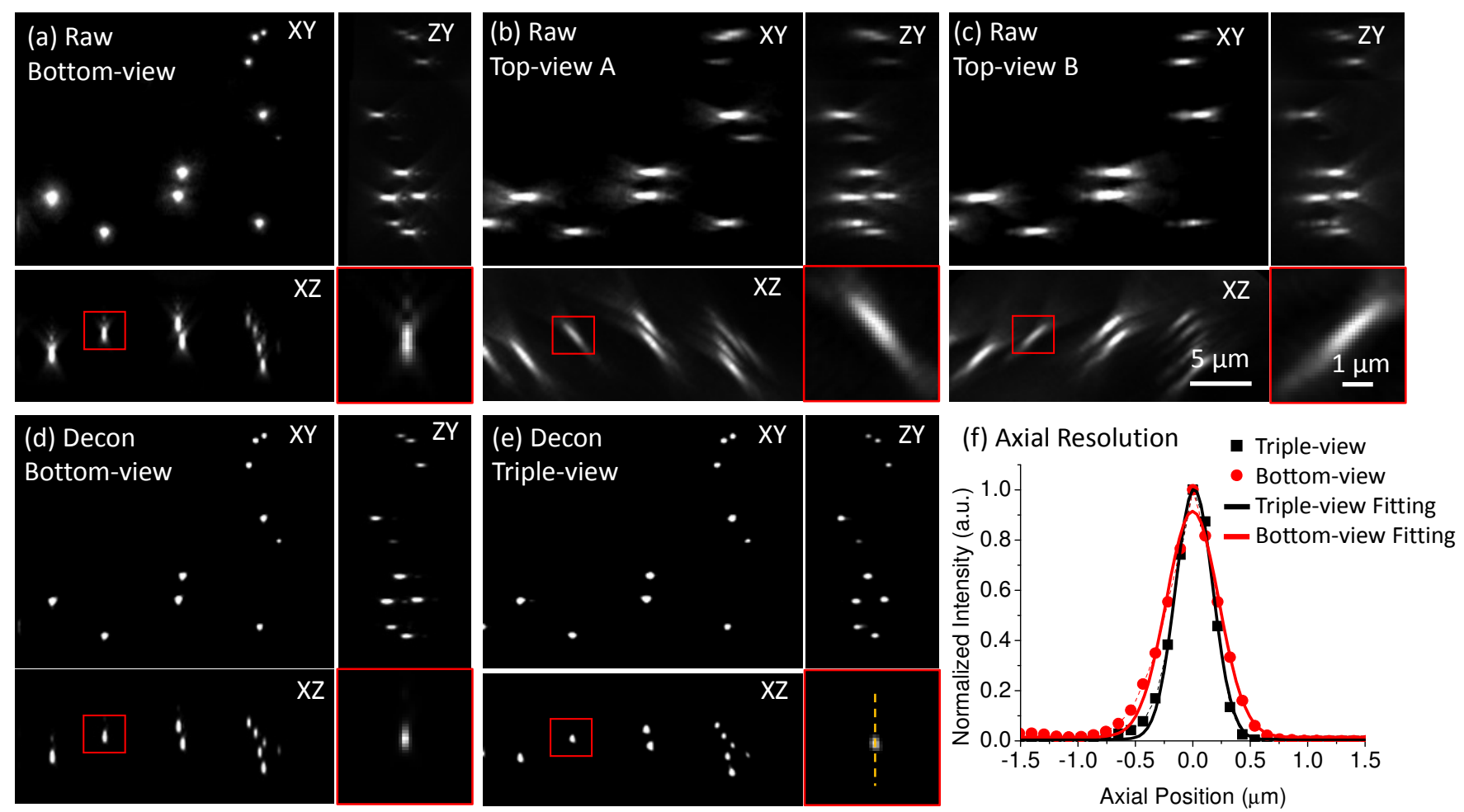

Fig. S2. Simultaneous triple-view widefield imaging improves axial resolution relative to single-view widefield imaging. a) Exemplary images of $100 \mathrm{~nm}$ fluorescent beads, as viewed in lateral (left) and axial (right, bottom) cross sections, captured from the lower objective (C). A higher magnification XZ view of in the indicated bead (red rectangle) is also shown at lower right. The same region is also shown as viewed through top objectives (b, c), after deconvolution of only the bottom view (d) and after registration and joint deconvolution of all three views (e). As indicated by axial line cuts through the bead (f), simultaneous triple-view acquisiton improves axial resolution $\sim 1.5$-fold (to $341 \pm 13 \mathrm{~nm}$ ) relative to single-view (539 $\pm 30 \mathrm{~nm}$ ) imaging, while maintaining lateral resolution (single view: $233 \pm 7 \mathrm{~nm}$, triple-view: $237 \pm 13 \mathrm{~nm}$ ). Dual-view deconvolution was performed over 60 iterations; tripleview over 180 iterations. 
(a)

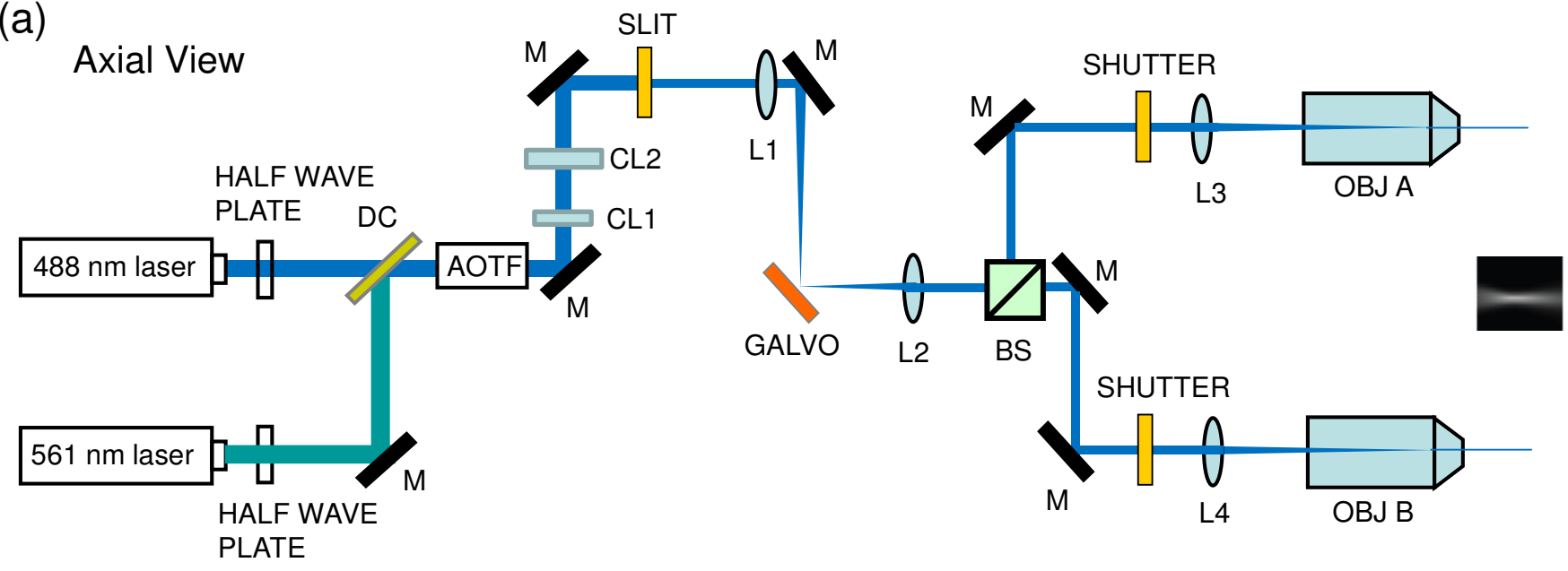

(b) Lateral View

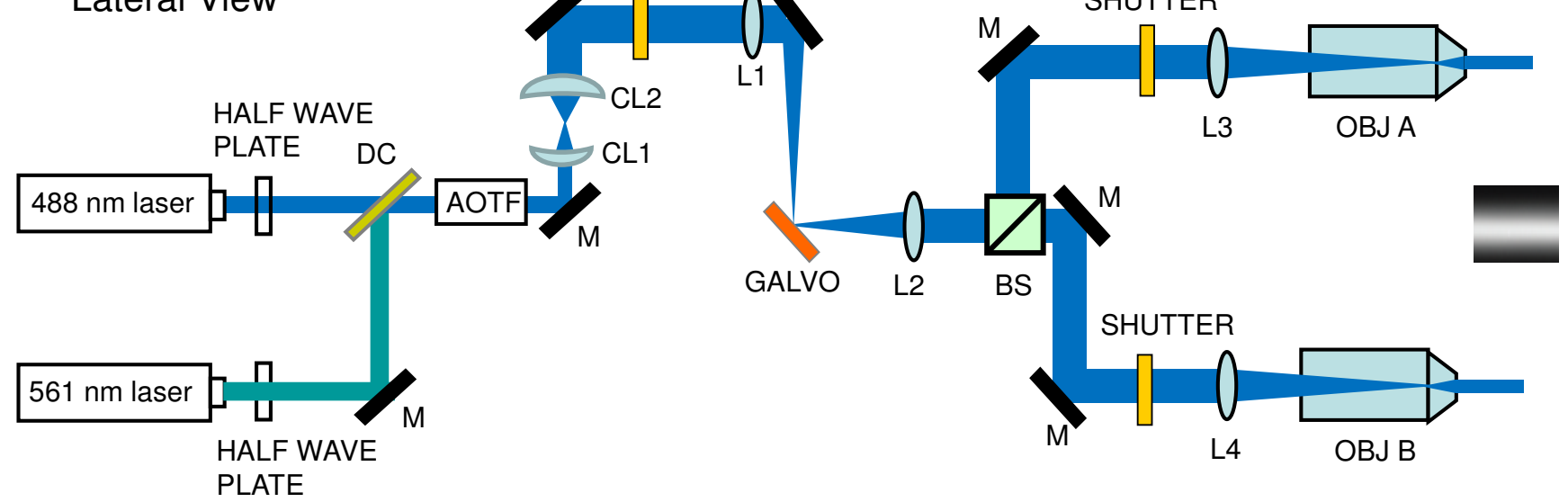

Fig. S3. Excitation optics used in upper objective path: (a) axial view and (b) lateral view. $488 \mathrm{~nm}$ and $561 \mathrm{~nm}$ lasers are combined via a dichroic mirror (DC). Both lasers are directed through half wave plates and an acoustic optical tunable filter (AOTF) for power control and shuttering. Cylindrical lens beam expander (CL1 and CL2) shape the excitation into a light sheet, and a rectangular slit (SLIT) further controls the thickness of the sheet. The light sheet is re-imaged to the sample via lens pairs L1 and L2, and L3 and OBJ A (objective A) for A view excitation, or L4 and OBJ B (objective B) for B view excitation, each in a 4f configuration. A beam-splitter cube (BS) and liquid-crystal shutters (SHUTTER) provide independent control of illumination in each SPIM arm. A galvanometric mirror (GALVO) is placed at the front focal plane of L2 and reimaged onto the back focal plane of OBJ A and OBJ B. During light sheet imaging, this GALVO is stationary, and the sample is moved through the stationary light sheet via an xy piezo stage. In wide-field imaging, this GALVO is scanned at $1 \mathrm{KHz}$ during each exposure $(10 \mathrm{~ms})$ over an angular range of \pm 0.2 degrees (mechanical), thereby translating the light sheet axially at the sample plane to create wide field excitation with cross-sectional area $\sim 80 \mu \mathrm{m}$ x $80 \mu \mathrm{m}$. Steering mirrors: rectangles marked 'M'. 


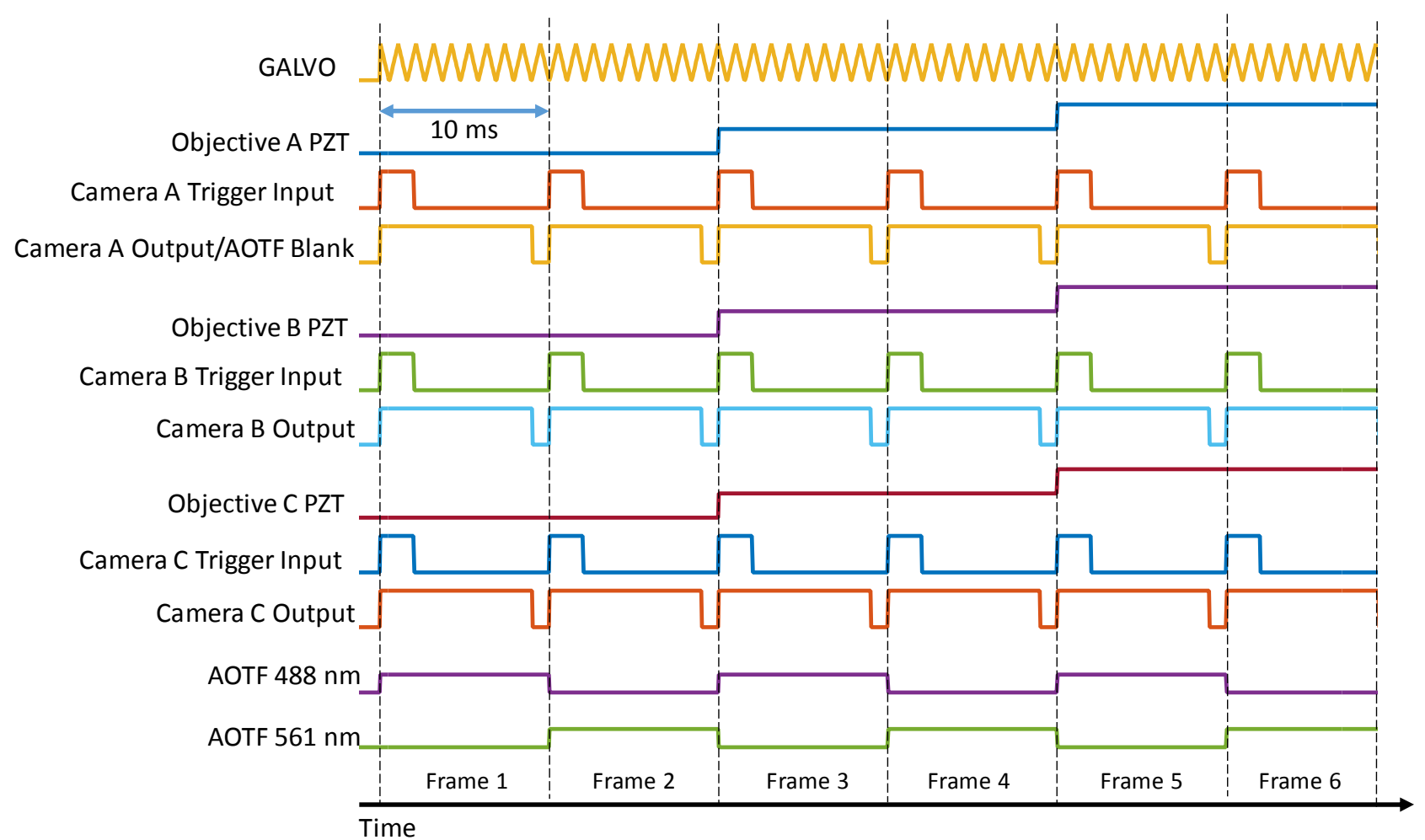

Fig. S4. Waveforms used in triple-view wide-field acquisition. Six (3 2-color) frames at three different positions at the sample are shown. PZT: piezoelectric transducer. 'A', 'B', 'C' refer to the objectives in Fig. 1. See Online Methods for more detail. 
(a) Wide field mode - lateral resolution

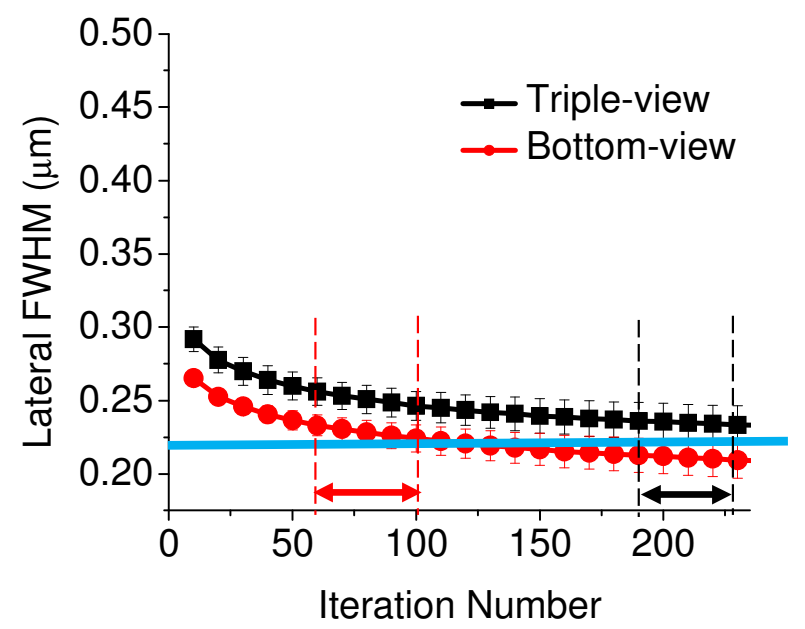

(b) Wide field mode - axial resolution

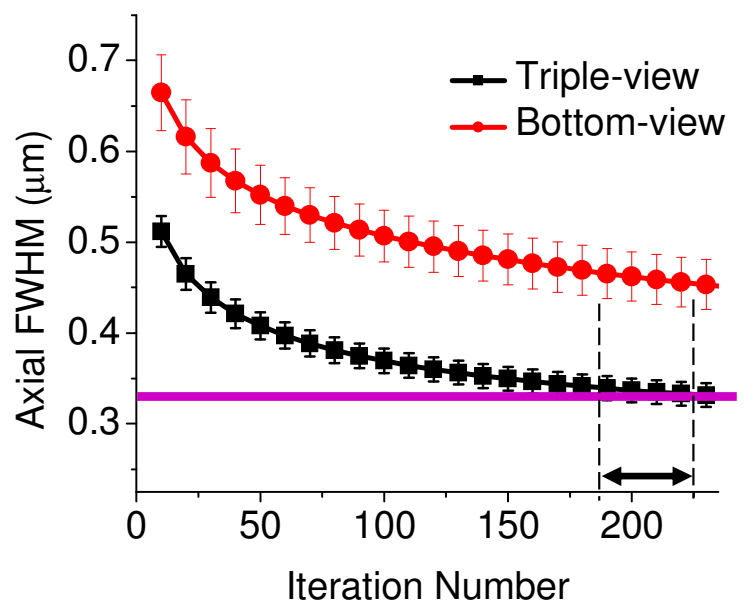

(c) Light sheet mode - lateral resolution

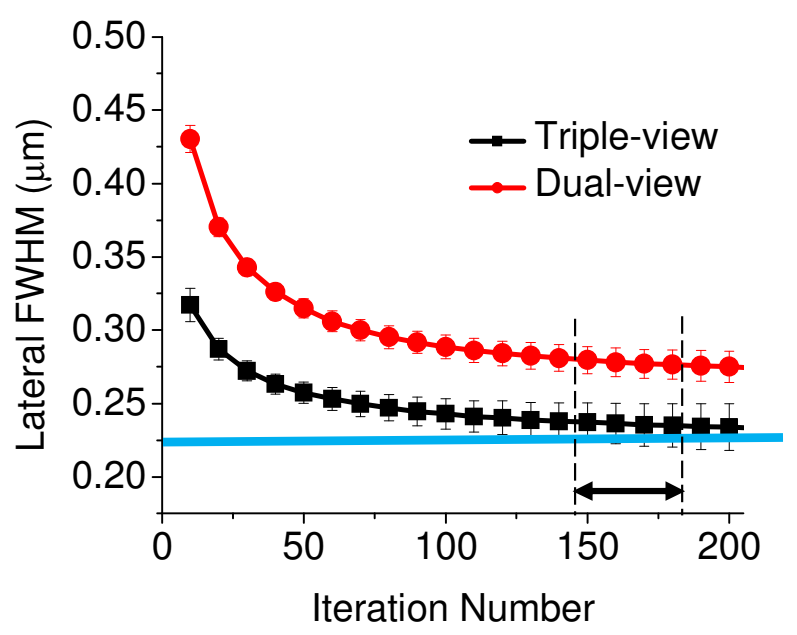

(d) Light sheet mode - axial resolution

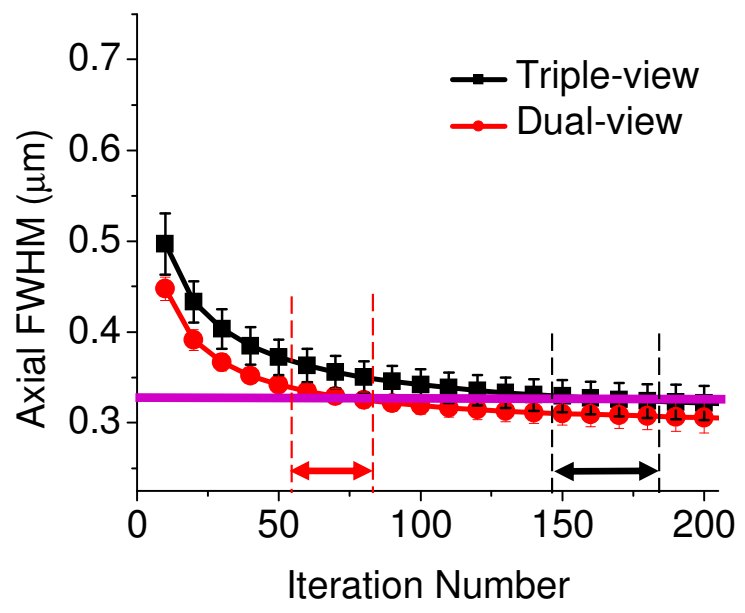

Fig. S5. Convergence of lateral and axial resolution when performing joint deconvolution in wide-field and light-sheet imaging. a) Lateral resolution after deconvolution in bottom wide-field (red) or joint deconvolution of all 3 wide-field views, as assayed by measuring the full width at half maximum (FWHM) of $10100 \mathrm{~nm}$ yellow-green fluorescent beads (means and standard deviations are shown). b) Same as a), but for light-sheet illumination; comparison is now between triple-view detection (via objectives A, B, C, black) and dual-view detection (objectives A, B). c)/d) Same as a)/b) but now comparing axial resolution. Blue lines in $\mathbf{a}, \mathbf{c}$ indicate the lateral resolution limit $\lambda /(2 * \mathrm{NA})$, calculated at $\lambda=525 \mathrm{~nm}, \mathrm{NA}=1.2$; purple lines in $\mathbf{b}$, $\mathbf{d}$ indicate the axial resolution limit, determined by the lateral resolution limit from the top views, i.e., $\lambda /(2 * \mathrm{NA}), \lambda=525 \mathrm{~nm}$, and $\mathrm{NA}=0.8$. Dotted lines and arrows indicate the range of cutoff iterations used in this paper. In general, triple-view imaging requires $\sim 3 \mathrm{x}$ the number of iterations required for dual-view imaging. 


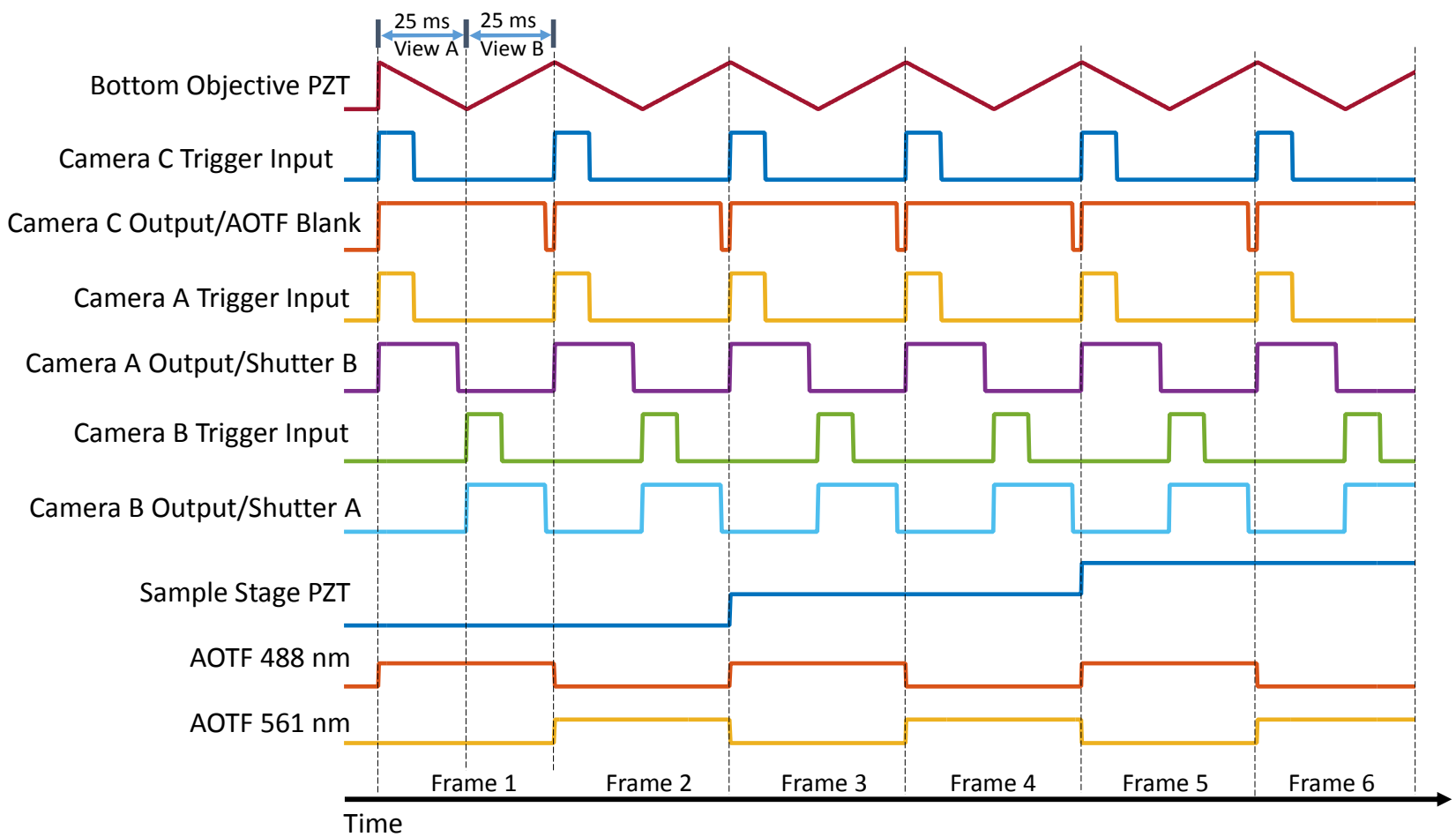

Fig. S6. Waveforms used in triple-view light-sheet acquisition. Six (3 2-color) frames at three different positions at the sample are shown. PZT: piezoelectric transducer. 'A', 'B', 'C' refer to the objectives in Fig. 1. See Online Methods for more detail. 
(a) Camera confocal slit mode timing diagram in one frame
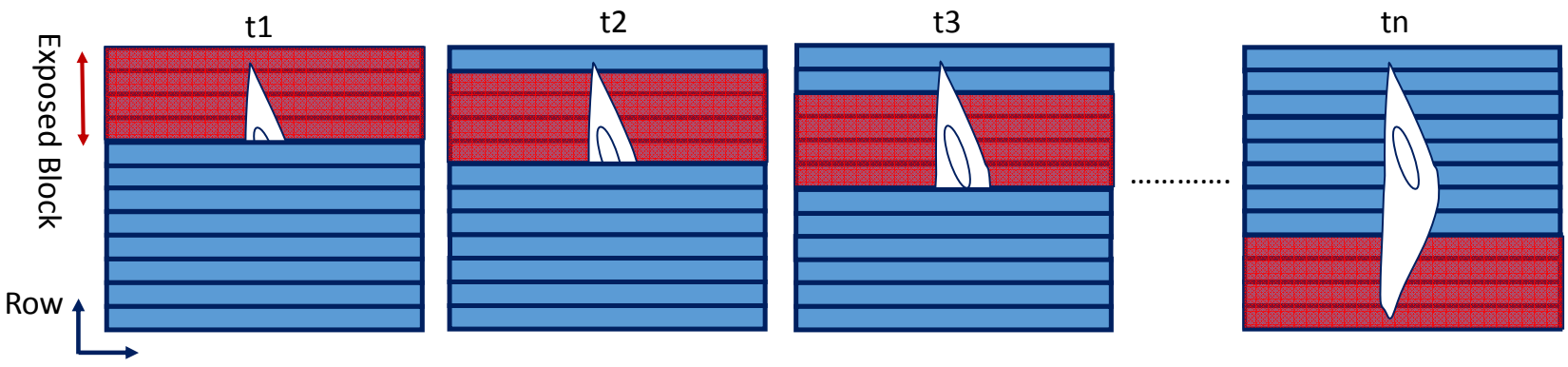

(b) Schematic of bottom-view acquisition
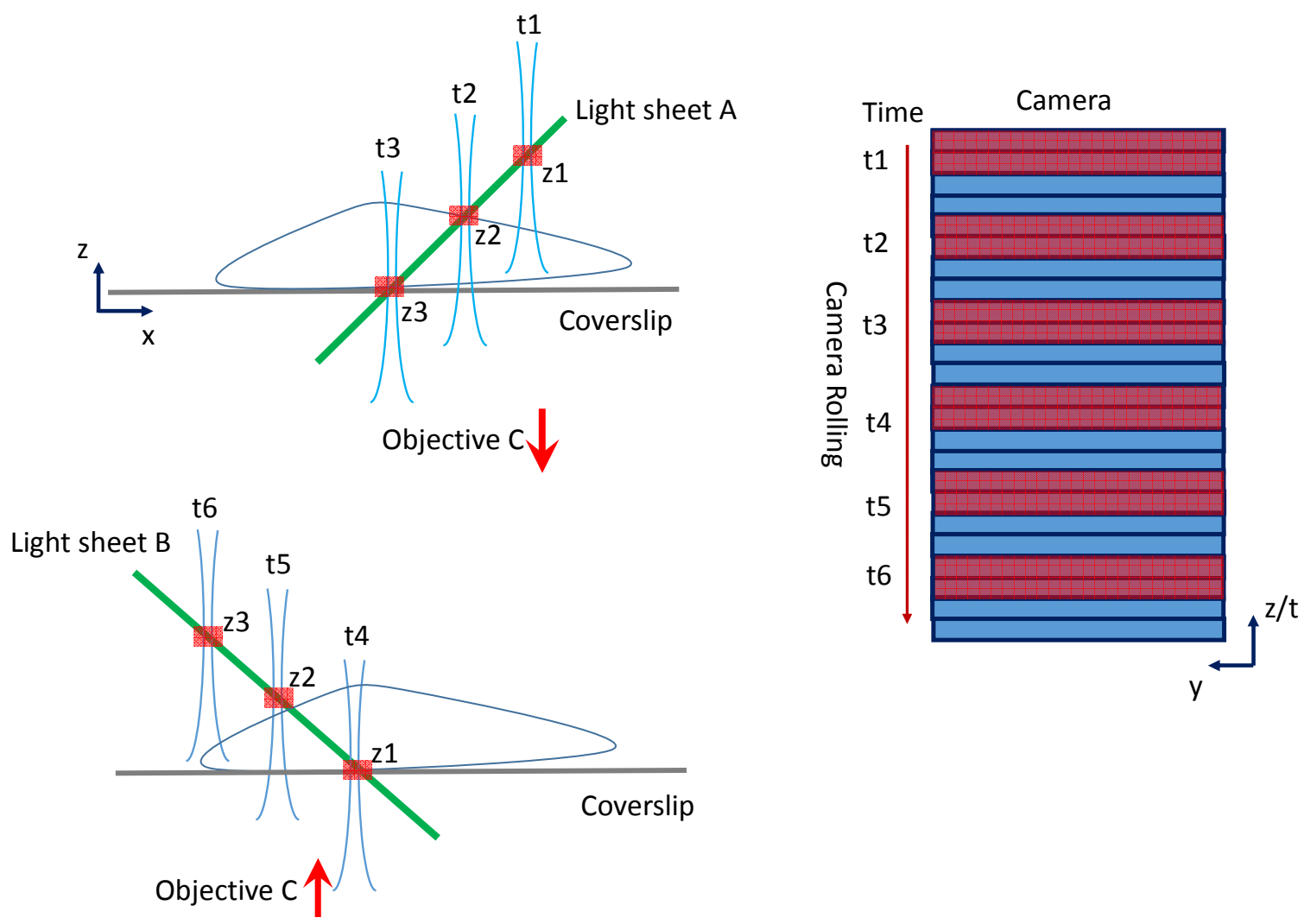

Fig. S7. Partially confocal imaging through objective C, during light-sheet illumination via objectives A/B. a) Schematic indicating serial capture of successive column blocks. At any instant, most of the detection pixels are masked, blocking out-of-focus light at these locations. The image is built up as the virtual slit 'rolls' along the camera, following the illuminated region. b) Schematic indicating relationship between illumination geometry, via objectives A/B, detection through objective C, detection pixels, time, and axial position in the sample. Red bars within light sheet indicate blocks of captured pixels at indicated axial position and time. 

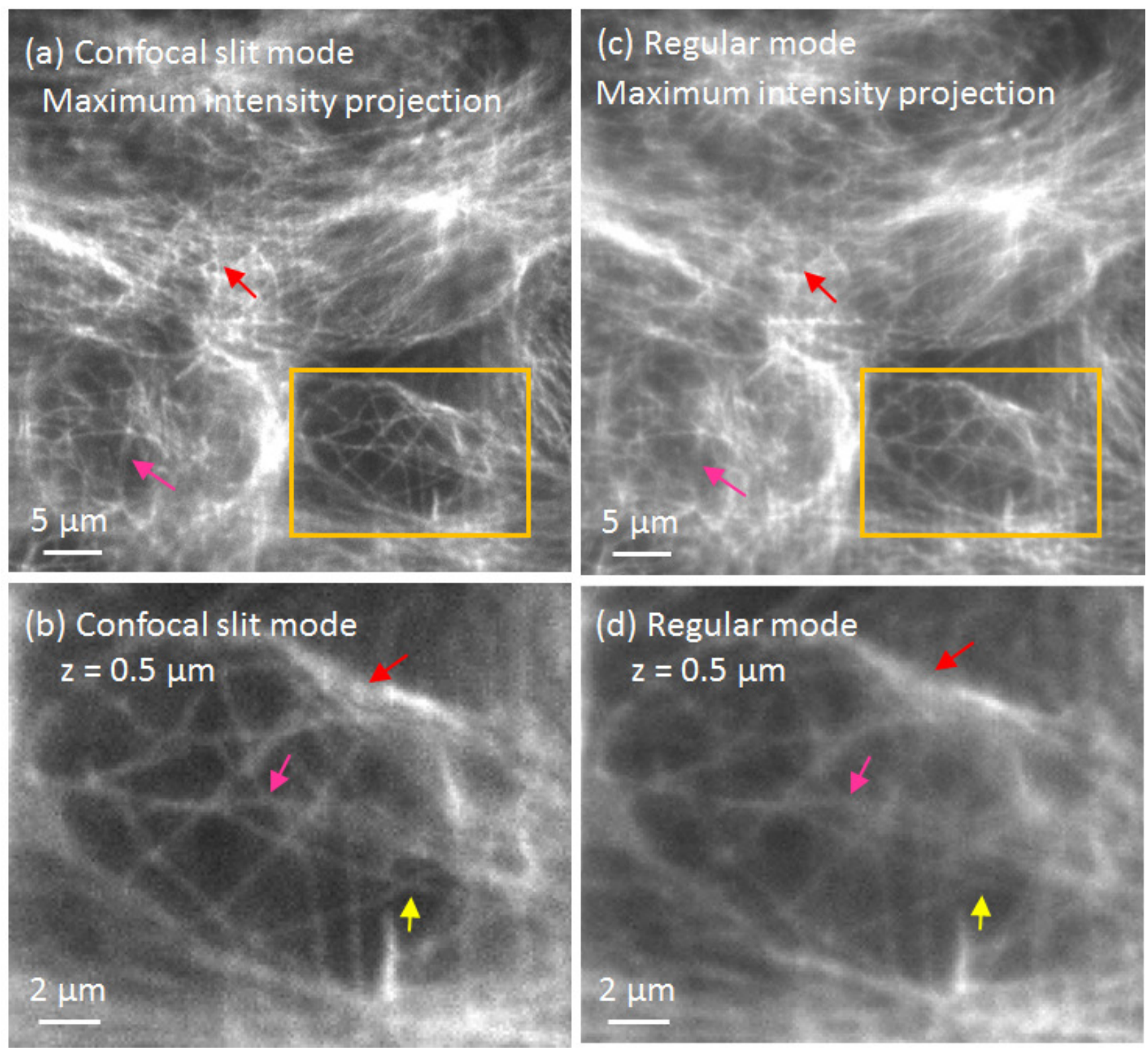

Fig. S8. Improvements in lower view (light-sheet mode) after piezo synchronization with rolling shutter detection. a) Maximum intensity projection from immunolabeled microtubules in fixed, U20S cell. Imaging stack was $3 \mu \mathrm{m}$ thick. Piezo synchronization with rolling shutter was applied. b) Same as a), but without rolling shutter. c) Higher magnification view derived from single slice $0.5 \mu \mathrm{m}$ from the coverslip, at location indicated by yellow rectangle in a). d) Higher magnification view derived from single slice $0.5 \mu \mathrm{m}$ from the coverslip, at location indicated by yellow rectangle in $\mathbf{c})$. The width of the slit in $\mathbf{a}, \mathbf{b}$ was set to 10 pixels $(1.08 \mu \mathrm{m})$. Note the improved contrast in $\mathbf{a}, \mathbf{b}$ compared to $\mathbf{c}, \mathbf{d}$ (colored arrows). 

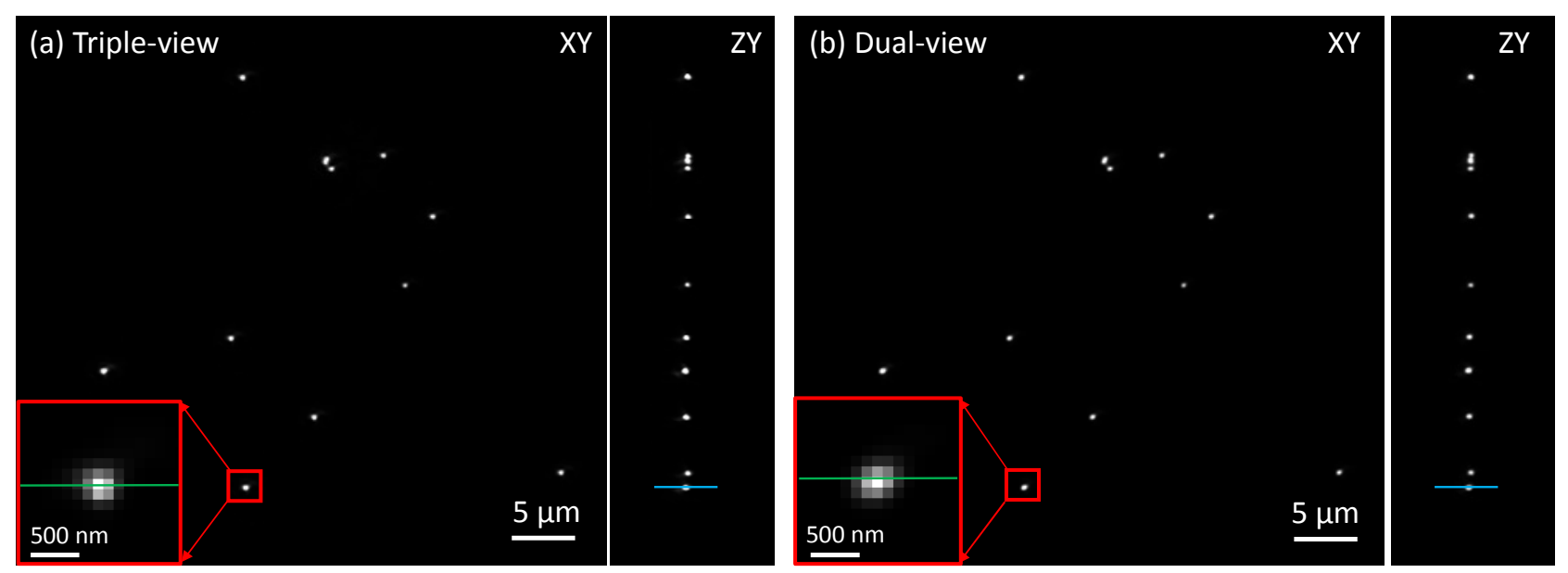

(c) Lateral profile

(d) Axial profile
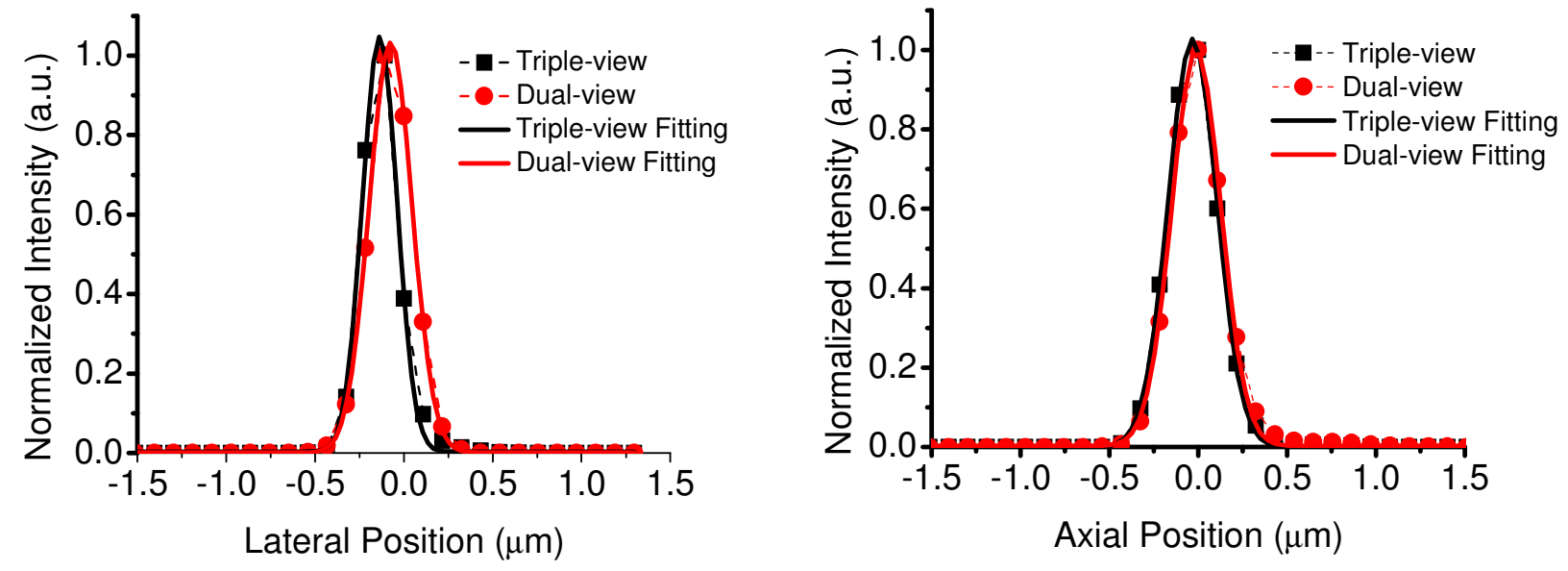

Fig. S9. Triple-view light sheet imaging improves lateral resolution relative to dual-view (diSPIM) imaging. a) Images of 100 $\mathrm{nm}$ yellow green fluorescent beads, as viewed after triple-view acquisition, registration, and fusion. Lateral (left) and axial (right) views are shown, as is a higher magnification view of a bead (inset). b) Same as a), but after dual-view (top two objectives only) acquisition, registration, and fusion. c) Exemplary line cuts through lateral bead insets in a, b, with Gaussian fits superposed. d) Same as c), but line cut is taken along axial direction as indicated in ZY views in a, b. Dual-view deconvolution was performed over 60 iterations; triple-view over 180 iterations. Lateral FWHM in dual-view: $306 \pm 7 \mathrm{~nm}$ and in triple-view $235 \pm 15 \mathrm{~nm}$. Axial FWHM in dual-view: $335 \pm 8 \mathrm{~nm}$ and in triple-view $324 \pm 19 \mathrm{~nm}$ (mean \pm standard deviation, $N=10$ beads). 
(a) Top-view Schematic

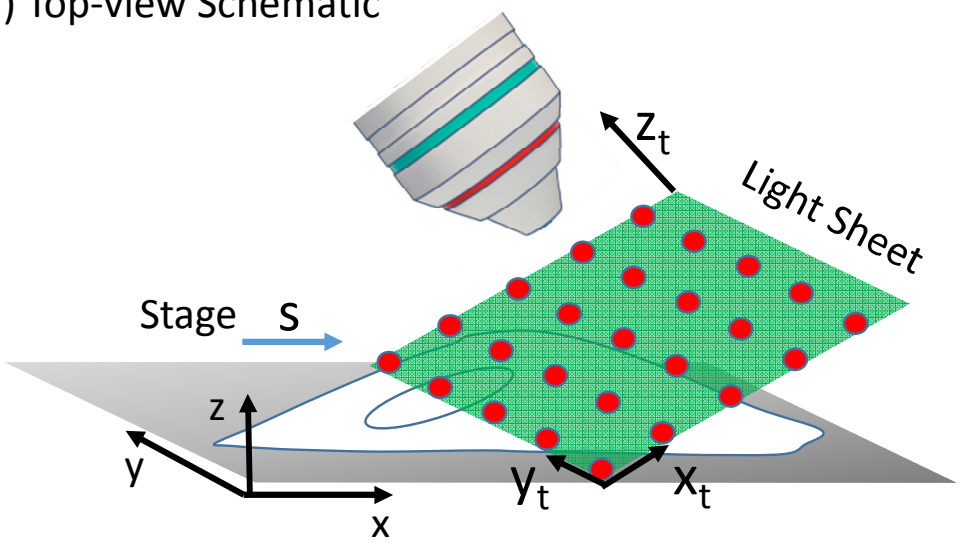

(b) Transformation Grid

- Measured pixels $\left(x_{t}, y_{t}, s\right)$

- Interpolated pixels along $x_{t}$

$\diamond$ Interpolated pixels along $z_{t}$

$$
\begin{aligned}
& x=x_{t} / \sqrt{2}+s \\
& z=x_{t} / \sqrt{2} \\
& y=y_{t}
\end{aligned}
$$

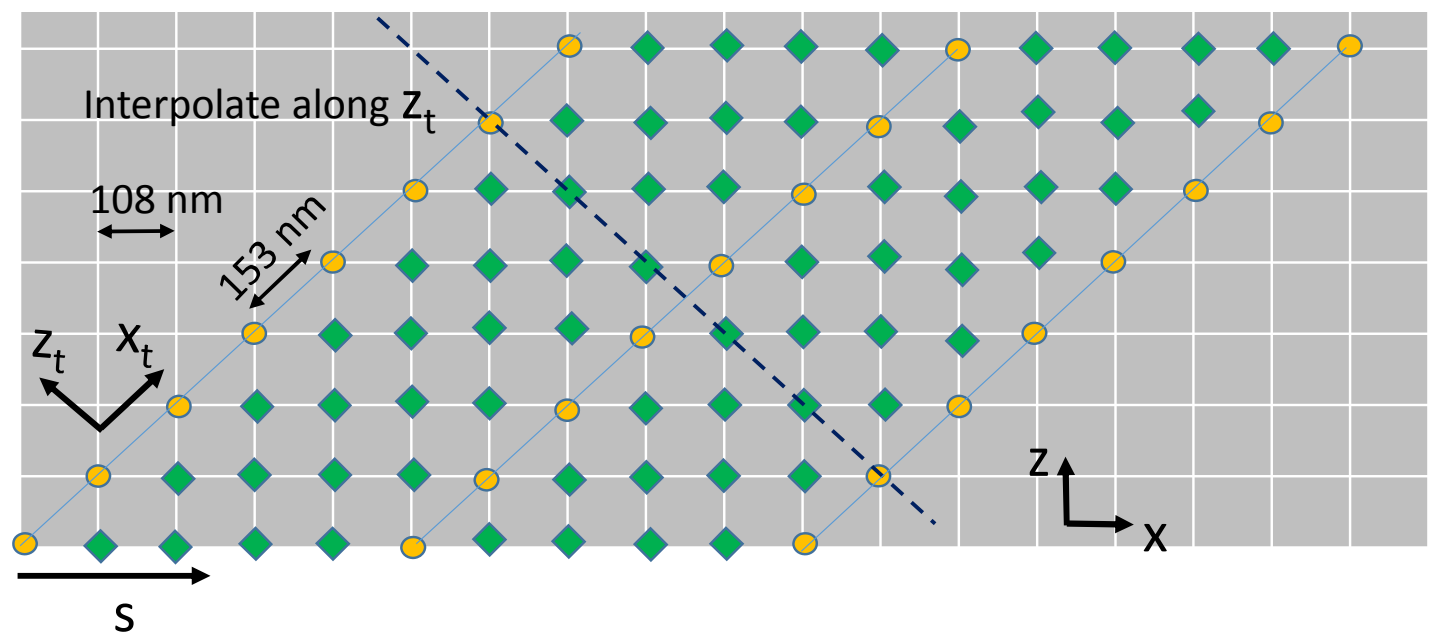

Fig. S10. Data transformation and interpolation from raw top to conventional (bottom) view. a) Schematic indicating top detection objective and associated coordinate system $\left(\mathrm{x}_{\mathrm{t}}, \mathrm{y}_{\mathrm{t}}, \mathrm{z}_{\mathrm{t}}\right)$, light sheet (green), cellular sample, coverslip, direction of stage-scan through light-sheet (s), and conventional coordinate system (x,y, z). b) Associated pixel grid indicating desired isotropic pixel dimensions $\left(108 \mathrm{~nm}\right.$ ), parallelogram of interpolated pixels (yellow dots along $\mathrm{xt}_{\mathrm{t}} /$ green diamonds along $\mathrm{zt}$ ) derived from measured pixels (red dots in a), axes in original top and desired conventional views, and interpolation axes (dotted line, $\mathrm{z}_{\mathrm{t}}$; blue line $\mathrm{x}_{\mathrm{t}}$ both at 45 degrees with respect to $\mathrm{z}$ and $\mathrm{x}$ axes). Also indicated are transformations that map interpolated pixels from top view to conventional bottom view. 
(a) Bottom-view Schematic

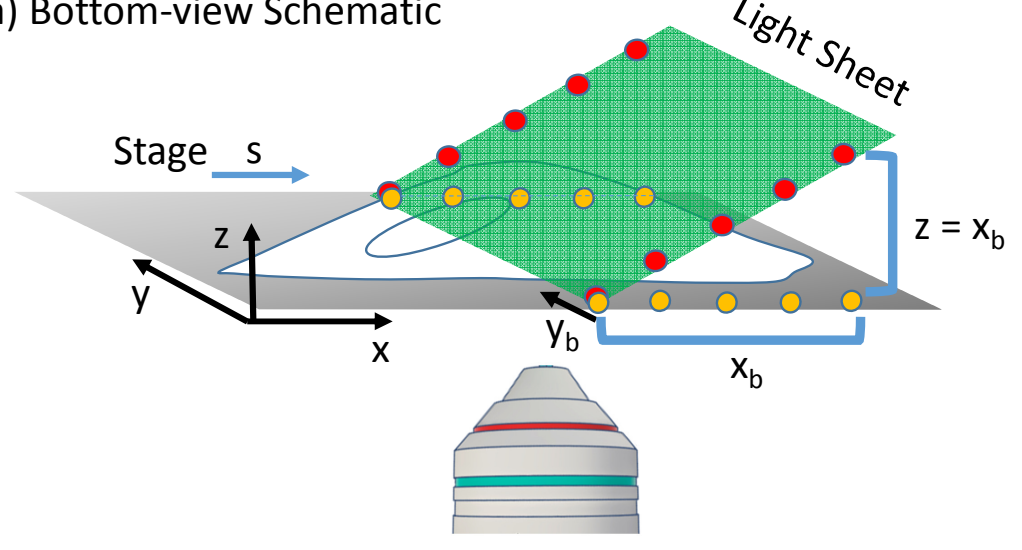

- Measured pixels $\left(x_{b}, y_{b}, s\right)$

- Projected pixels Interpolated pixels along z

(b) Transformation Grid

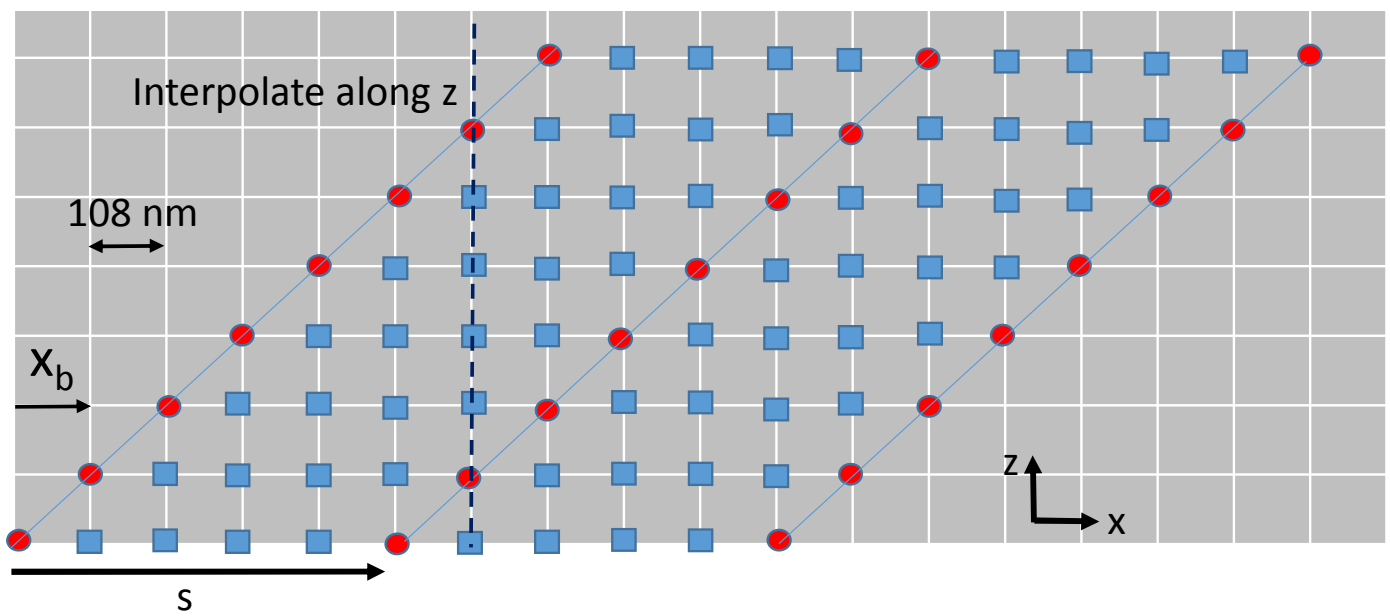

Fig. S11. Data transformation and interpolation from raw bottom to conventional (bottom) view. a) Schematic indicating bottom detection objective and associated coordinate system ( $\mathrm{xb}, \mathrm{yb}$ ), light sheet (green), cellular sample, coverslip, direction of stagescan through light-sheet (s), and conventional coordinate system (x,y, z). b) Associated pixel grid indicating desired isotropic pixel dimensions $(108 \mathrm{~nm})$, parallelogram of projected pixels (i.e. axial information projected along the $\mathrm{xb}_{\mathrm{b}}$ axis) interpolated pixels (blue squares along $\mathrm{z}$ axis) derived from measured (yellow dots in a) and projected pixels (red dots in a, b) after transformation, relevant axes in original bottom and desired conventional views, and interpolation axis (dotted line, $\mathrm{z}$ ). Also indicated are transformations that map interpolated pixels from raw bottom view to conventional bottom view. 

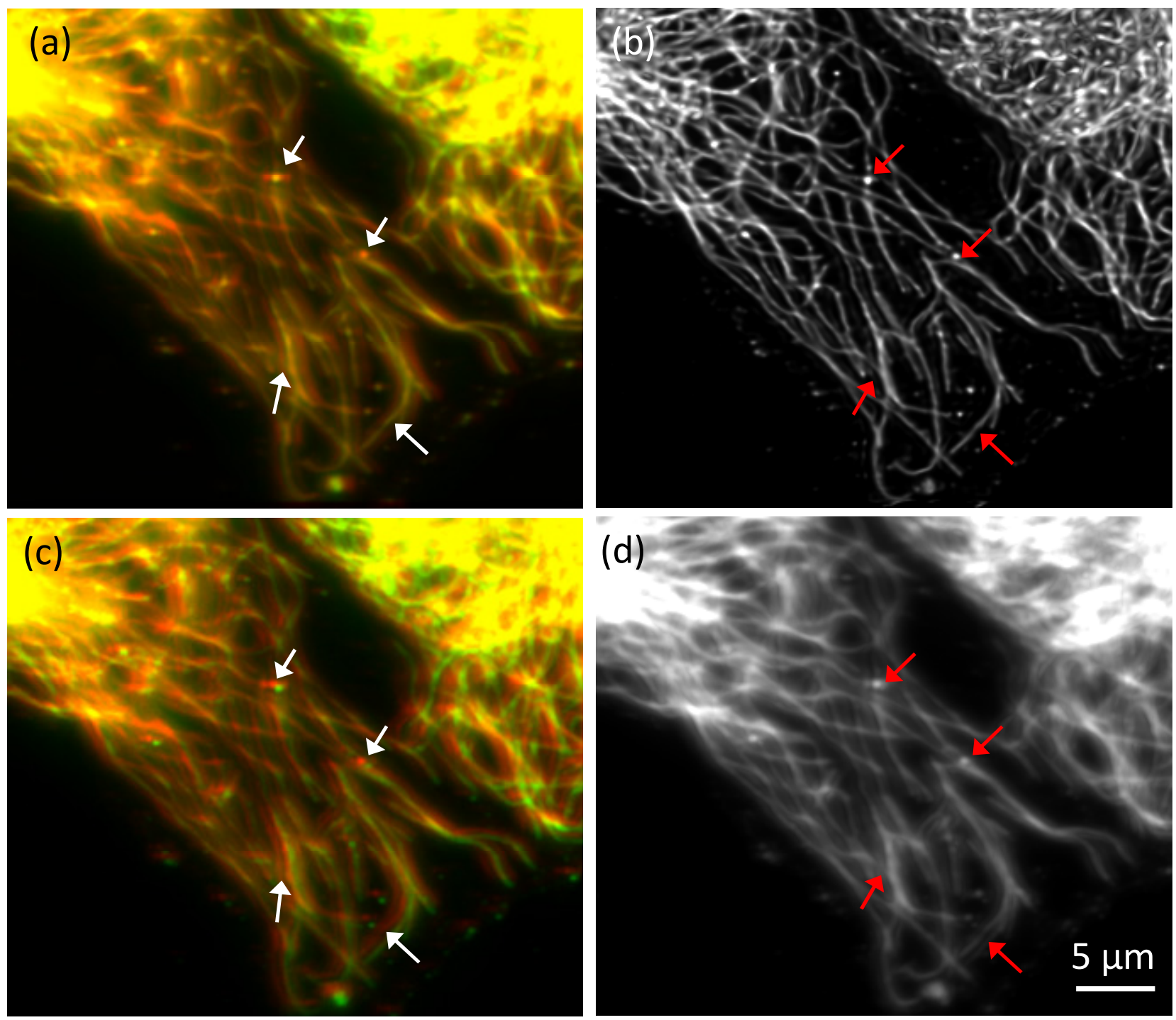

Fig. S12. Examples of good and bad registrations in light-sheet mode, and corresponding deconvolutions: a) good registration result obtained by using registration matrix derived from individually deconvolved views that is subsequently applied to the raw views; b) corresponding deconvolution; c) bad registration result obtained when using registration matrix directly derived from raw views; d) corresponding deconvolution. XY maximal intensity projections are shown, a top view is colored in red, and the bottom view in green. Arrows highlight comparative regions of good (top) or bad (bottom) registration, and correspondingly effective (b) or ineffective (d) deconvolution. 

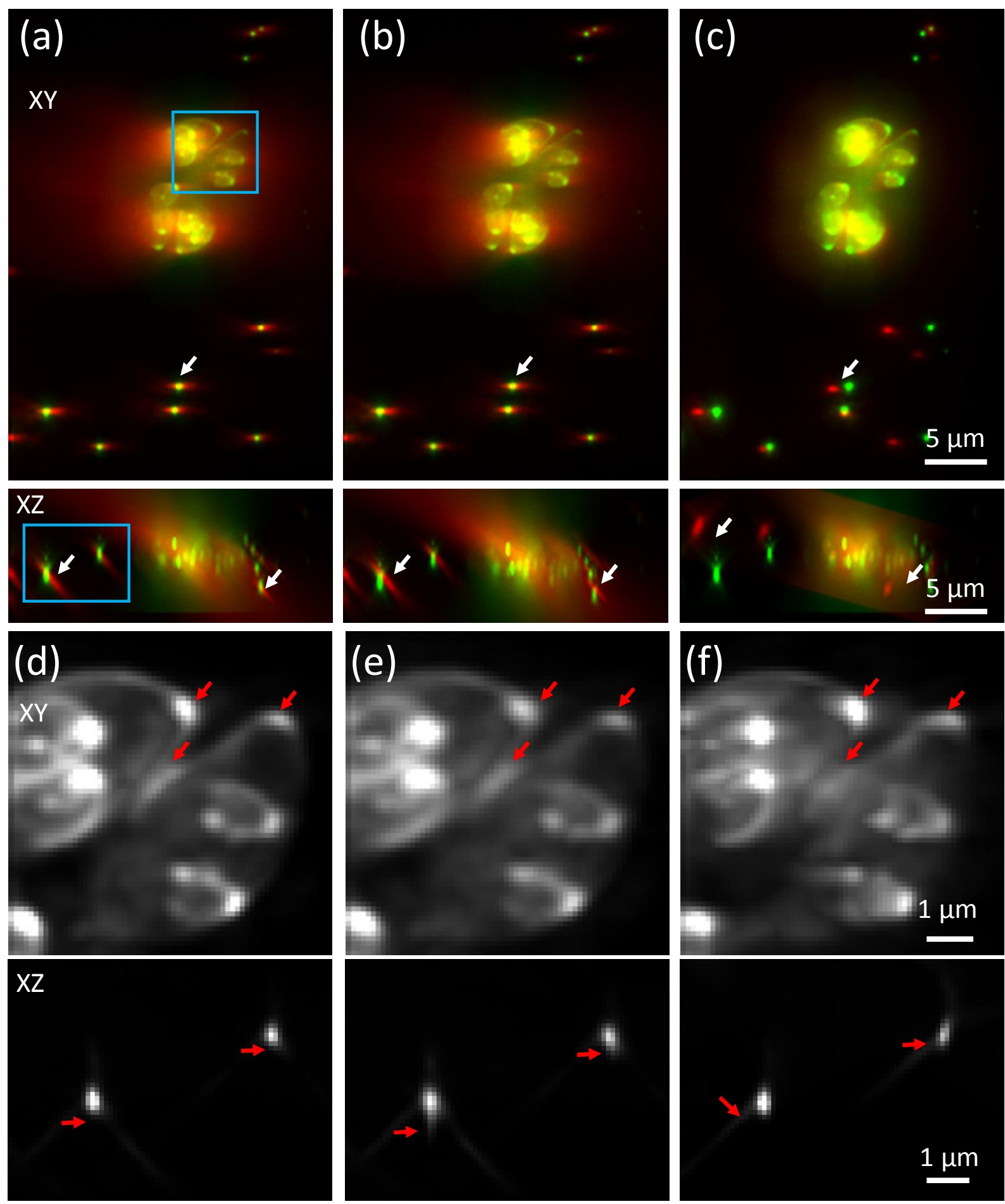

Fig. S13. Registration accuracy in wide-field mode: (a) An example of a good registration result, obtained by using deconvolved views and beads; (b) bad registration outcome by using deconvolved views, but artificially masking beads; (c) bad registration result with direct registration on raw data. $\mathrm{XY}$ and $\mathrm{XZ}$ are maximum intensity projections, one of the two top views is colored in red, and the bottom view is colored in green. (d-f): Corresponding deconvolution results based on (a-c) showing higher magnification views of the blue rectangles in $\mathrm{XY} / \mathrm{XZ}$ projections indicated in (a). Red arrows highlight features in good (d) and progressively worse deconvolution $(\mathbf{e}, \mathbf{f})$ outcomes. 
Additive Deconvolution

(a)

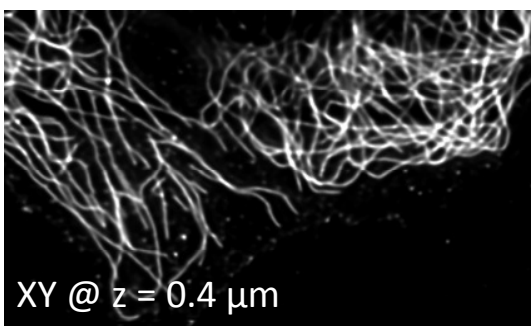

(b)

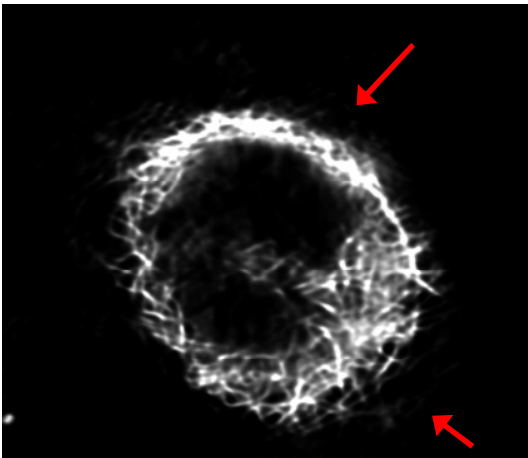

$\mathrm{XY} @ \mathrm{z}=1.6 \mu \mathrm{m}$

(c)

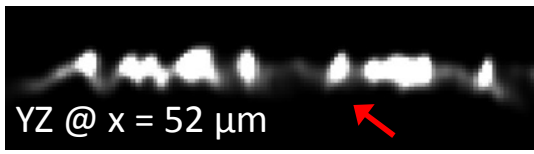

(d)

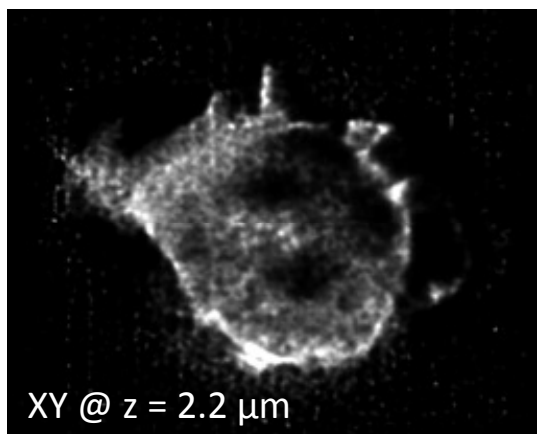

Multiplicative Deconvolution
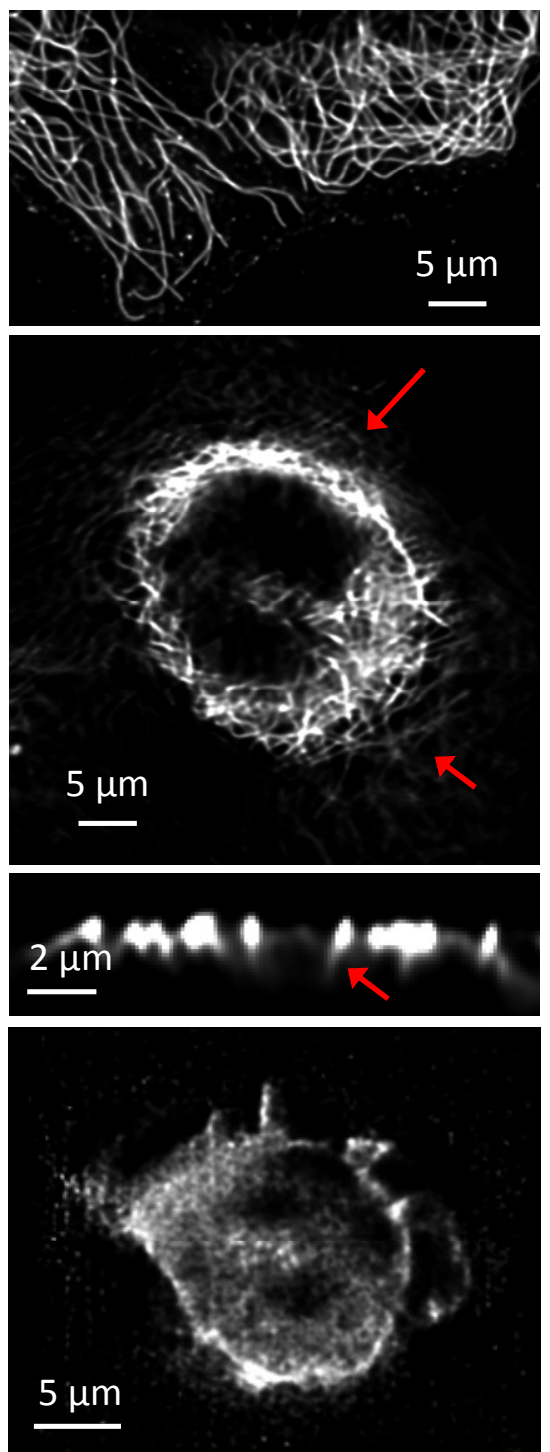

Alternating Deconvolution


Fig. S14. Comparison of three different deconvolution methods. (a-c) Fixed microtubules in a U2OS cell, as viewed after additive (left column), multiplicative (middle column) or alternating (right column) joint deconvolution. XY images are similar near coverslip, at high signal levels (a), but additive deconvolution provides better sectioning as revealed farther from the coverslip (b) or in axial profiles (c). Arrows indicate comparative regions far from the coverslip or in axial profiles. Furthermore, when SNR is low (d) as in these images of stably transfected GFP- actin in T cells, noise is amplified more when using multiplicative or alternating deconvolution. 
(a)

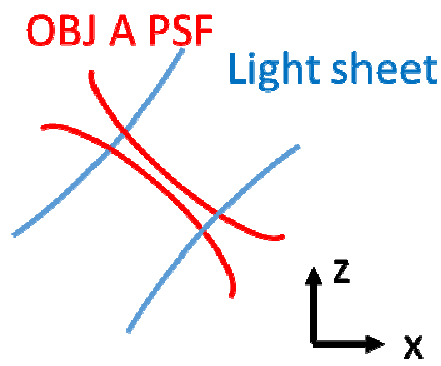

PSFA $_{\text {sheet }}=$

Light-sheet X PSFA widefield

(b)

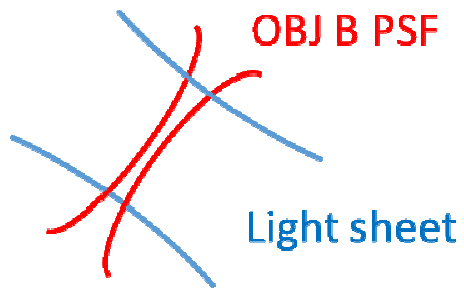

$$
\begin{aligned}
& \text { PSFB }_{\text {sheet }}= \\
& \text { Light-sheet X } \text { PSFB }_{\text {widefield }}
\end{aligned}
$$
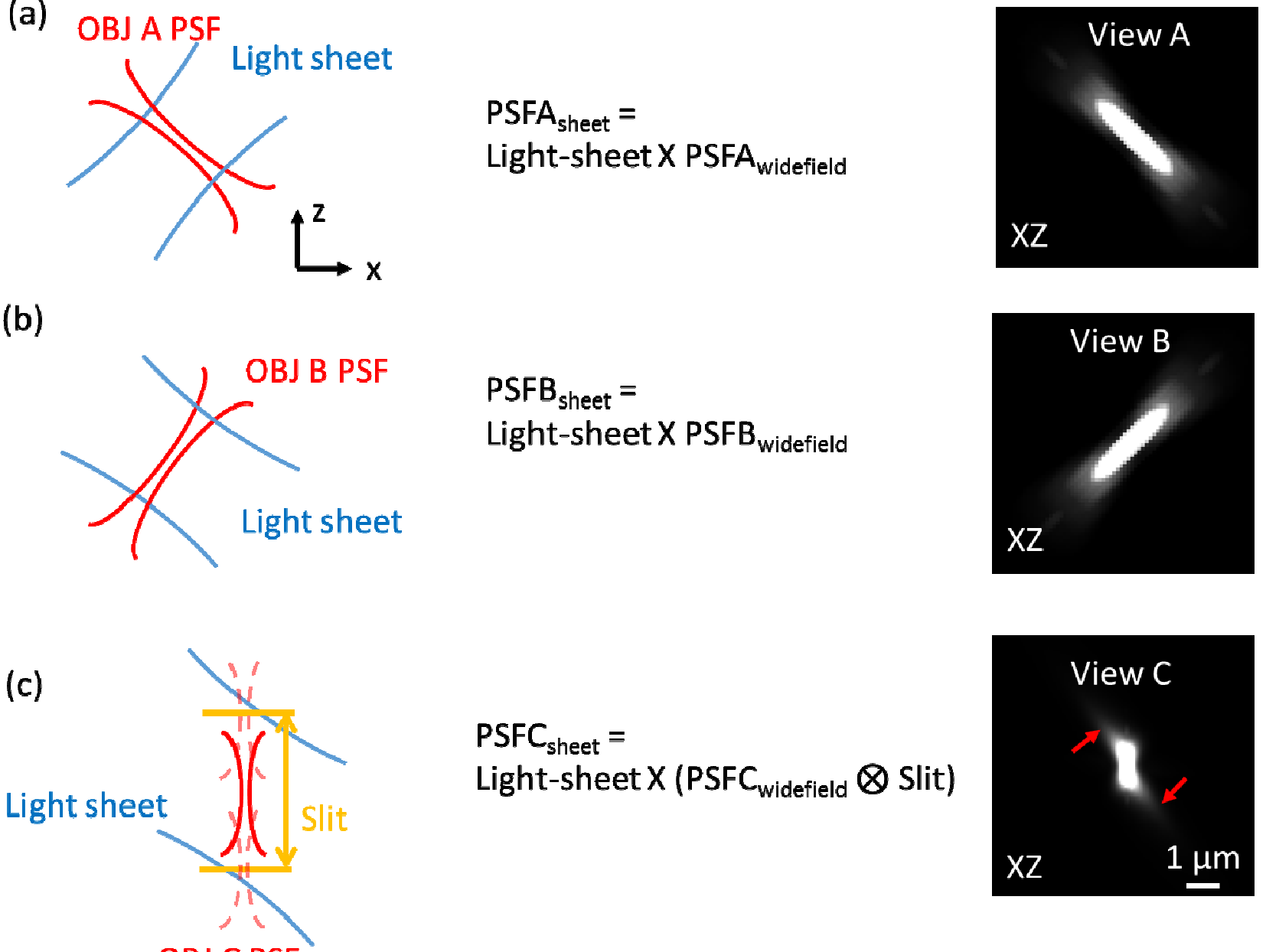

OBJ C PSF

Fig. S15. Modeling the PSFs when using light-sheet mode. The point spread functions of the two upper view (imaged by OBJ A/B, a, b) are given by the product of light sheet illumination and wide-field detection PSF. For the lower view, the detection PSF of the lower objective (OBJ C, c) is given by the wide-field detection PSF convolved with the 'rolling slit' function. Since the width of the slit is $1 \mu \mathrm{m}$, and the lower objective is at a different axial position as the slit traverses the camera, we axially shift the PSF over $1 \mu \mathrm{m}$ and average the result. Finally, we multiply this shifted, averaged detection PSF with the light sheet to generate the lower view PSF. Note the rotation of $\mathbf{a}, \mathbf{b}$ with respect to the lower view coordinate system, and the 'wings' (red arrows, $\mathbf{c}$ ) in the lower view PSF make the Gaussian function a poor choice for modeling any of the PSFs. 


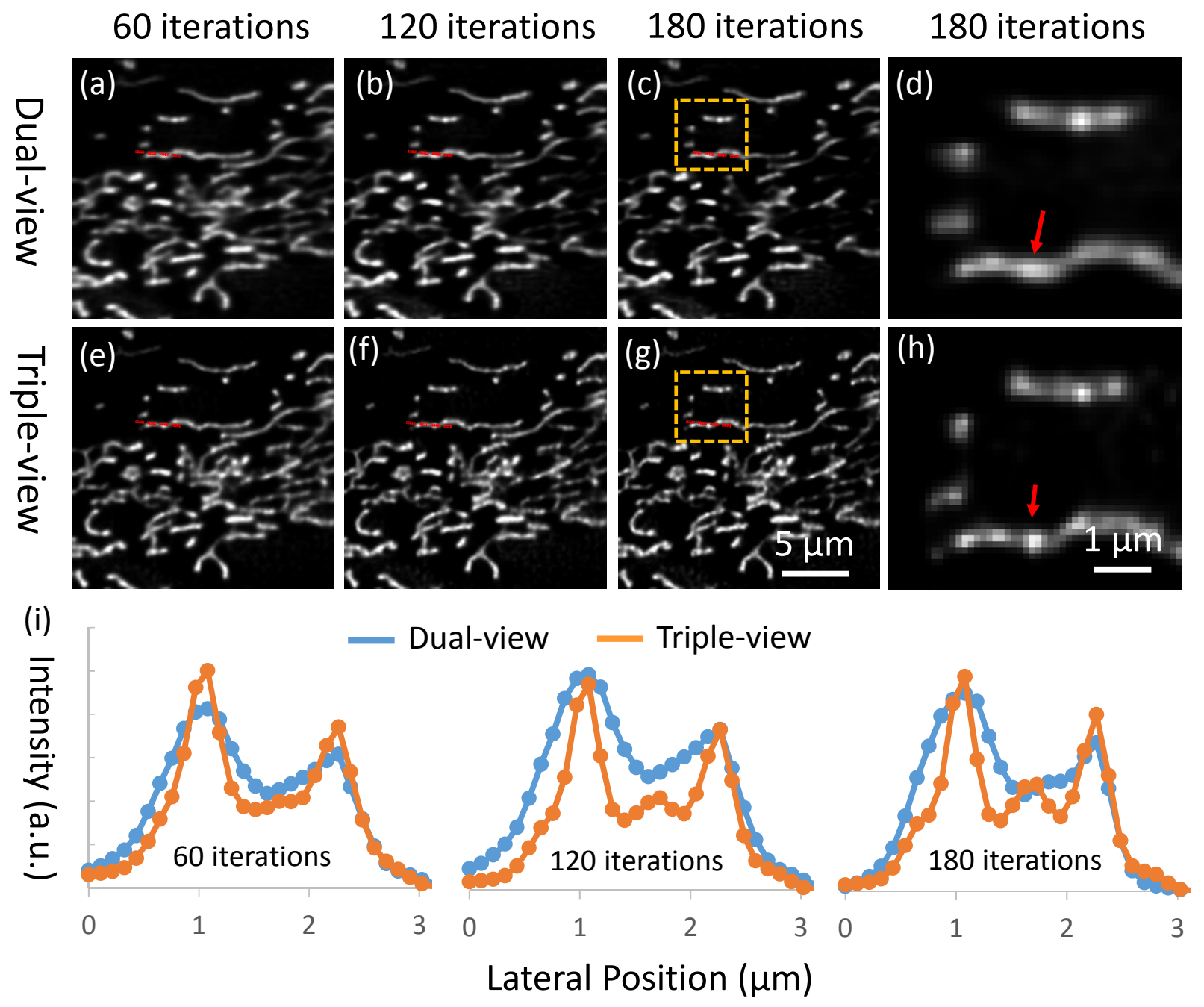

Fig. S16. Triple-view imaging produces sharper imaging than dual-view, regardless of iteration number. Stained mitochondria were imaged in dual-view (a-c) and triple-view (e-g) light-sheet configurations and deconvolved using 60 (a, e), 120 (b, f), and 180 (c-d, g-h) iterations. d, h: Higher magnification views of yellow rectangle in $\mathbf{c}$, $\mathbf{g}$, emphasizing greater resolution of triple-view imaging. As confirmed by inspection (red arrows, $\mathbf{d}, \mathbf{h}$ ) and line profiles (i, corresponding to red lines in a-c, e-g), triple-view imaging provides better resolution than dual-view imaging, regardless of iteration number. 